\title{
Lúpus Eritematoso Sistêmico: Prevenção da Doença Aterosclerótica Coronariana
}

\section{Systemic Lupus Eritemathosus: prevention of coronary atherosclerosis}

\author{
Renata Carneiro de Menezes ${ }^{(1)}$, Maria de Fátima Carneiro de Menezes ${ }^{(2)}$
}

\begin{abstract}
A sobrevida de pacientes com lúpus eritematoso sistêmico (LES) tem aumentado significativamente nas últimas décadas, deixando de ser uma doença de curso rápido e fatal para agora apresentar-se como uma condição mais benigna e de curso crônico. Com a mudança na história natural do LES, resultante do diagnóstico e tratamento precoces, terapia criteriosa e melhor controle das infecções, novos problemas têm surgido nesses pacientes, entre os quais a aterosclerose. As evidências mais recentes reconhecem a aterosclerose como uma causa importante de morbidade e mortalidade no LES e exigem intervenções direcionadas a esse problema. A avaliação de fatores de risco não é realizada de rotina nesses pacientes e muitos desconhecem o risco elevado de doença cardiovascular no LES. Considerando a faixa etária das pacientes envolvidas, argumenta-se que o LES seja considerado condição de alto risco para aterosclerose, à semelhança de pacientes com diabetes mellitus. As estatinas, amplamente utilizadas na terapia de dislipidemias, têm sido também avaliadas em pacientes com LES, por seu papel potencial no controle da inflamação e resposta imune. Estudos clínicos randomizados controlados avaliando a eficácia de estatinas na proteção contra eventos coronarianos no LES estão em andamento. Entretanto, há evidências da eficácia de estatinas na melhora da disfunção endotelial nesses pacientes, evento inicial na aterogênese e preditor de evento cardiovascular em várias populações. Esta seção apresenta alguns artigos recentes que discutem a terapia com estatinas, o uso potencial da aspirina e outras medidas na abordagem da aterosclerose em LES.
\end{abstract}

Bruce IN. Cardiovascular disease in lupus patients: should all patients be treated with statins and aspirin? (Doença cardiovascular em pacientes lúpicos: todos os pacientes devem ser tratados com estatinas e aspirina?). Best Pract Res Clin Rheumatol 19(5): 823-38, 2005. University of Manchester, Rheumatism Research Centre and Manchester Children's University Hospitals Trust, Manchester, Reino Unido.

Neste artigo de revisão, o autor propõe uma abordagem pró-ativa no manejo dos fatores de risco convencionais, com "alvos ideais" baseados na observação do LES como uma condição predisponente para aterosclerose. As orientações propostas para avaliação do colesterol e indicações para estatinas em pacientes lúpicos são as seguintes: ${ }^{*} \mathrm{c}-\mathrm{LDL}$ colesterol $<100 \mathrm{mg} / \mathrm{dl}$, com revisão do perfil lipídico ao menos anualmente; *se c-LDL $=100-130 \mathrm{mg} / \mathrm{dl}$ : encorajar a redução de peso e modificação dietética, rever a dose do corticosteróide e considerar droga antimalárica (AM); *Terapia medicamentosa (estatinas) deve ser considerada quando LDL > $130 \mathrm{mg} / \mathrm{dl}$ com ou sem outros fatores de risco (FRs), ou quando persistentemente $>100 \mathrm{mg} / \mathrm{dl}$, mesmo após as modificações anteriores. Em relação à profilaxia com aspirina, na população geral é considerada em pacientes com evento vascular prévio e na prevenção primária em pacientes com risco elevado de doença aterosclerótica cardiovascular (DAC).

1. Mestra em Medicina Interna pela Universidade Federal de Pernambuco (UFPE) e Reumatologista do Hospital Oscar Coutinho - Instituto Materno-Infantil Professor Fernando Figueira (IMIP).

2. Professora adjunta da Disciplina de Reumatologia da UFPE e Coordenadora do Programa de Residência Médica em Reumatologia do Hospital das Clínicas da UFPE.

Endereço para correspondência: Renata Carneiro de Menezes. Rua Frei Matias Teves, 280, sl 510, Recife, Pe, e-mail: menezesrc@bol.com.br 
O autor orienta o uso de aspirina em pacientes com LES que, na ausência de contra-indicação, preencham um dos seguintes critérios: *História prévia de infarto do miocárdio (IM), angina, acidente vascular cerebral (AVC) ou ataque isquêmico transitório (AIT); * presença de anticorpos antifosfolipídeos (aFLS); *hipertensão; *DM; *hipercolesterolemia; *tabagismo. Neste contexto, uma proporção significativa de pacientes lúpicos (aproximadamente 30\%) irá requerer estatinas e, visto que $88 \%$ preenchem ao menos uma indicação para o uso profilático de aspirina, a norma seria a sua utilização de rotina no LES na ausência de contra-indicação de uso.
Outras medidas adicionais são também recomendadas, como o controle adequado da atividade da doença, a consideração dos AMs como adjuntos, tendo em vista sua segurança relativa, a dos inibidores da enzima conversora de angiotensina (IECAs) no controle da pressão arterial ou da disfunção do ventrículo esquerdo e o controle do peso corporal.

$\mathrm{O}$ autor conclui o artigo enfatizando a necessidade de ensaios clínicos bem conduzidos para o avanço, além dessas recomendações iniciais.

Petri M, Kiani AN, Post W, Magder L. Lupus Atherosclerosis Prevention Study (LAPS): a randomized double blind placebo controlled trial of atorvastatin versus placebo. [Lupus Atherosclerosis Prevention Study (LAPS): Um ensaio randomizado duplo-cego placebo-controlado de atorvastatina versus placebo]. Arthritis Rheum 54 (Suppl): S520, 2006. Johns Hopkins University e University of Maryland, Baltimore, Estados Unidos.

O LAPS é um ensaio clínico com duração de dois anos de atorvastatina $v s$. placebo desenhado a partir da hipótese de que as estatinas podem modificar tanto o componente inflamatório quanto o lipídico da aterosclerose no LES. Os dados foram apresentados no ACR 2006. Estudaram-se 200 pacientes ( $92 \%$ do sexo feminino, com idade média de 44 \pm 11 anos) com o objetivo de avaliar os efeitos da atorvastatina na aterosclerose subclínica em pacientes lúpicos. Os pacientes foram randomizados para receber atorvastatina $40 \mathrm{mg} /$ dia ou placebo, sendo vistos após um mês e a cada três meses por dois anos.

Os critérios de exclusão incluíram história de doença cardíaca coronariana, níveis de c-LDL > $190 \mathrm{mg} / \mathrm{dl}$ ou triglicerídeos $>500 \mathrm{mg} / \mathrm{dl}$. Utilizaram-se como medidas de avaliação, no início do estudo e ao final dos dois anos, o cálcio coronário (CC) por tomografia computadorizada (TC), o espessamento médio-intimal (EMI) da artéria carótida comum e a atividade de doença pelo SLEDAI. Os resultados não mostraram diferença significativa entre os grupos atorvastatina e placebo na progressão do CC nem na atividade da doença.
Em relação ao EMI carotídeo, houve aumento em ambos os grupos $(\mathrm{p}<0,0001)$, mas sem significância estatística $(\mathrm{p}=0,22)$. Houve, porém, uma diferença significativa na proporção de pacientes nos quais o EMI melhorou, permaneceu o mesmo ou piorou, favorecendo a atorvastatina $(\mathrm{p}=0,01)$. Hipertensão e idade foram os maiores preditores de EMI, enquanto a PCR foi um dos melhores preditores de CC, sugerindo que ateroscleroses carotídea e coronária podem ser diferentes no LES. Os eventos adversos mais comuns incluíram elevação de transaminases ( $7 \%$ em ambos os grupos) e creatinofosfoquinase ( $4 \%$ no grupo atorvastatina e $8 \%$ no placebo), reversível com a suspensão da droga e maior no LES do que o esperado na população geral.

Embora ainda haja um ano de estudo até que os resultados sejam disponibilizados, os dados de segurança mostram até o momento mais toxicidade da estatina, sugerindo atenção e necessidade de monitorização caso estatinas sejam iniciadas em um paciente lúpico. Os autores concluem que houve alguma evidência de que a atorvastatina retardou a progressão do EMI da carótida e que, considerando o risco aumentado de aterosclerose no LES, a terapia com estatina deve ser mais bem estudada nesses pacientes. 
Ferreira GA, Sato EI, Andrade LC, Telles RW, Navarro TP. Atorvastatin therapy improves endothelial-dependent vasodilation in systemic lupus erythematosus patients (Terapia com estatina melhora a vasodilatação endotélio-dependente em pacientes com LES). Ann Rheum Dis 66 (Suppl II): 461, 2007. Rheumatology Division, Hospital das Clínicas - Universidade Federal de Minas Gerais, Belo Horizonte, Rheumatology Division, Universidade Federal de São Paulo - Escola Paulista de Medicina, São Paulo, Brasil.

Este foi um ensaio clínico de intervenção realizado no Brasil e apresentado recentemente no EULAR. Pacientes com LES apresentam redução da vasodilatação endotéliodependente e evidências demonstram que as estatinas são capazes de melhorar a função endotelial independentemente do seu efeito na redução dos lipídeos. O objetivo foi avaliar a eficácia da atorvastatina na melhora da vasodilatação endotéliodependente em pacientes lúpicos com e sem fatores de risco convencionais para doença cardíaca coronariana (DCC). Sessenta e quatro pacientes lúpicas (idade média $31 \pm 8$ anos) receberam atorvastatina $20 \mathrm{mg} /$ dia durante 8 semanas; 31 pacientes neste grupo de intervenção não apresentavam FRs convencionais para DCC, enquanto 33 outras apresentavam HAS e/ou dislipidemia e/ou obesidade. Vinte e quatro pacientes lúpicas controle (idade média $34 \pm 7,5$ anos) não receberam a droga e foram observadas pelo mesmo período.

Utilizou-se ultra-som de alta resolução para medir o diâmetro da artéria braquial no repouso, durante hipere- mia reativa e após nitrato sublingual, no início do estudo e ao final das oito semanas. Atorvastatina esteve associada a aumento significativo da dilatação mediada pelo fluxo (DMF) ( $\mathrm{p}<0,001)$, enquanto a dilatação mediada pelo gliceriltrinitrato (DGTN) não foi alterada $(\mathrm{p}=0,514)$. Observou-se aumento da DMF nas pacientes com $(\mathrm{p}=0,046)$ e também naquelas sem FRs clássicos para DCC $(\mathrm{p}=0,001)$. $\mathrm{O}$ diâmetro da artéria braquial no repouso também melhorou significativamente nas que receberam atorvastatina $(\mathrm{p}<0,001)$.

Não se observou diferença significativa do diâmetro arterial nem da DMF nas pacientes controles ao final do estudo. Quando comparado ao controle, o tratamento com atorvastatina esteve associado a um aumento significativo do diâmetro de repouso $(\mathrm{p}<0,001)$ e da DMF $(\mathrm{p}=0,009)$. Os autores demonstraram que a atorvastatina foi eficaz na melhora da função endotelial nas pacientes lúpicas, independentemente da presença de FRs convencionais para DCC.

Riboldi P, Gerosa M, Meroni PL. Statins and autoimmune diseases (Estatinas e doenças auto-imunes). Lupus 14(9): 765-8, 2005. Allergy, Clinical Immunology Rheumatology Unit, IRCCS Istituto Auxologico Italiano, Department of Internal Medicine, University of Milan, Milão, Itália.

Este artigo de revisão se concentra nos mecanismos antiinflamatórios e imunomoduladores das estatinas, demonstrados em modelos experimentais in vitro e in vivo, fornecendo novos conhecimentos para o uso das estatinas no tratamento de doenças auto-imunes sistêmicas.

Além do já bem estabelecido efeito na redução dos lipídeos, os inibidores da 3-hidróxi-3-metilgluteril(HMG)CoA redutase apresentam efeitos pleiotrópicos antiinflamatórios, antitrombóticos e atividade estabilizadora de placa por meio de vários mecanismos, com ação na inflamação, hemóstase, resposta imune e atividade antiaterosclerótica. Entre esses efeitos estão a inibição da indução da expressão de moléculas do complexo maior de histocompatibilidade classe II (MHC-II) pelo interferon-gama, reduzindo a ativação subseqüente de células $\mathrm{T}$, a redução da deposição glomerular de imunoglobulinas e dos níveis de anti-DNAds e de PCR, a melhora da função endotelial lipídeo-independente, o desvio no balanço trombótico/fibrinolítico favorecendo este último e propriedades antioxidantes, como a modulação dos níveis de nitrotirosina, um marcador de 
dano protéico oxidativo pelos oxidantes derivados do óxido nítrico e preditor independente de risco aterosclerótico.

Visto que em adição aos fatores de risco tradicionais, inflamação sistêmica, respostas imunemediadas e trombofilia têm um papel importante na patogênese da aterosclerose prematura em doenças auto-imunes como
LES, as estatinas têm sido propostas como agente ideal na redução dos lipídeos nesses pacientes. Apesar das evidências experimentais, sua eficácia na incidência de mortes relacionadas a infarto do miocárdio ou doença coronariana nessa população de pacientes deve ser mais bem estudada.

Ardoin SP, Sandborg C, Schanberg LE. Management of dyslipidemia in children and adolescents with systemic lupus erythematosus (Abordagem da dislipidemia em crianças e adolescentes com lúpus eritematoso sistêmico). Lupus 16 (8): 618-26, 2007. Department of Pediatrics, Duke University Medical Center, Durham, Carolina do Norte e Stanford University School of Medicine, Stanford, Califórnia, Estados Unidos.

Neste artigo de revisão, os autores comentam que o LES, fator de risco independente para aterosclerose, põe crianças e adolescentes lúpicos em grande risco para o desenvolvimento de seqüelas cardiovasculares, incluindo infarto do miocárdio (IM), na idade adulta. Dislipidemia e outros fatores de risco cardíacos tradicionais ocorrem freqüentemente no LES pediátrico e são subdiagnosticados e subtratados.

Para os autores, a triagem de rotina para a dislipidemia com perfil lipídico encontra-se indicada para crianças e adolescentes com LES. Caso os níveis das lipoproteínas estejam anormais, terapia de primeira linha envolve intervenções com dieta e exercício, por no mínimo seis meses. Para a dislipidemia persistente, os autores comentam sobre as diversas terapias farmacológicas disponíveis. Hidroxicloroquina, comumente utilizada no tratamento do LES, pode melhorar o perfil lipídico e deve ser considerada em todos os pacientes lúpicos. Estatinas e quelantes de ácidos biliares são tipicamente adicionados primeiro à dislipidemia, enquanto niacina e fibratos são reservados à doença refratária e prescritos numa abordagem multidisciplinar.

Os autores concluem que pesquisas futuras são necessárias no intuito de melhor esclarecer os mecanismos da dislipidemia no LES pediátrico, com ensaios clínicos bem desenhados para determinar as intervenções mais seguras e mais eficazes na correção do perfil lipídico e prevenção da aterosclerose nessa população.

Ruiz-Irastorza G, Egurbide MV, Pijoan JI, Garmendia M, Villar I, Martinez-Berriotxoa A, Erdozain JG, Aguirre C. Effect of antimalarials on thrombosis and survival in patients with systemic lupus erythematosus (Efeito dos antimaláricos na trombose e sobrevida de pacientes com lúpus eritematoso sistêmico). Lupus 15(9): 577-83, 2006. Department of Internal Medicine, Hospital de Cruces, University of the Basque Country, Bizkaia, Espanha.

Após décadas de uso empírico, vários ensaios clínicos randomizados controlados têm demonstrado a eficácia das drogas AMs na atividade no LES. Seus efeitos benéficos vão mais além, com estudos observacionais sugerindo propriedades antitrombóticas e na redução dos lipídeos.

O objetivo desta coorte foi investigar se os AMs protegem contra trombose e influenciam na sobrevida de pacientes com LES. Estudaram-se 232 pacientes lúpicos incluídos no estudo, no momento do diagnóstico e acompanhados a cada três meses, ou de acordo com a necessidade. A positividade para aFL foi definida segundo os critérios de Sapporo. Os desfechos foram trombose documentada (trombose venosa profunda, embolia pulmonar, trombose arterial periférica e visceral, IM, acidente vascular cerebral e ataque isquêmico transitório) e morte por qualquer motivo dentro do período de seguimento, que durou em média $10,6 \pm 7$ anos. A maioria ( $88 \%$ ) era mulher e $64 \%$ receberam AM durante qualquer período do estudo, com mediana do tempo de uso de 52 meses (3-228).

$\mathrm{O}$ uso de AM protegeu contra trombose (OR 0,28, IC de $95 \%, 0,08-0,90)$, enquanto a positividade para aFL (OR 3,16, IC de 95\% 1,45-6,88) e trombose prévia (OR 3,85 , IC de 95\%, 1,50-9,91) aumentou o risco de eventos trombóticos. Vinte e três pacientes faleceram, $19(83 \%)$ 
dos quais nunca haviam recebido AM. Não se observou morte por complicações CVs em nenhuma paciente tratada com AM. A taxa cumulativa de sobrevida em 15 anos foi de 0,68 para as que nunca $v s .0,95$ para as que em algum momento foram tratadas $(\mathrm{p}<0,001)$.
O estudo demonstrou a proteção dos AMs contra trombose e maior sobrevida dos pacientes em uso dessa droga. Diante dos achados e do seu excelente perfil de segurança, os autores defendem a utilização de rotina dos AMs nos pacientes com LES.

Erkan D, Harrison MJ, Levy R, Peterson M, Petri M, Sammaritano L, Unalp-Arida A, Vilela V, Yazici Y, Lockshin. Aspirin for primary thrombosis prevention in the antiphospholipid syndrome: a randomized, double-blind, placebo-controlled trial in asymptomatic antiphospholipid antibody-positive individuals (Aspirina na prevenção primária da trombose na síndrome antifosfolípide: um ensaio clínico randomizado, duplo-cego, placebo-controlado em indivíduos assintomáticos com anticorpo antifosfolípide positivo). Arthritis Rheum 56(7): 2382-91, 2007. Hospital for Special Surgery, Weill Medical College of Cornell University, New York, Estados Unidos.

Associados à inflamação crônica, pacientes com LES freqüentemente apresentam anticoagulante lúpico (ACL) ou anticorpos antifosfolipídeos (aFLs) e existem evidências de que alguns aFL possam ser pró-aterogênicos e prótrombóticos, sendo possíveis marcadores de risco para eventos cardiovasculares futuros.

O APLASA (Antiphospholipid Antibody Acetylsalicylic Acid) foi um ensaio clínico multicêntrico, randomizado, placebo-controlado no qual 98 indivíduos assintomáticos com aPL persistentemente positivo receberam aspirina $81 \mathrm{mg} /$ dia $(\mathrm{n}=48)$ ou placebo $(\mathrm{n}=40)$ com o objetivo de determinar a eficácia da intervenção na prevenção de trombose primária.

Em um estudo paralelo e observacional, 74 indivíduos com as mesmas características em uso de aspirina ou que não foram inclusos na randomização foram seguidos.
O tempo de seguimento foi de $2,3 \pm 0,95$ anos no APLASA e de 2,46 $\pm 0,76$ anos no estudo observacional. $O$ uso de aspirina não protegeu contra a incidência de trombose aguda $[2,75 / 100$ pacientes/ano no grupo da aspirina $v s$. $0 / 100$ pacientes/ano no placebo (OR 1,04 , IC de $95 \%$ $0,69-1,56, \mathrm{p}=0,83)]$. De maneira semelhante, no estudo paralelo também não se observou proteção.

Todos os pacientes com trombose, exceto um, apresentavam fatores de risco concomitantes e/ou doença auto-imune sistêmica no momento do evento. Os resultados sugerem que indivíduos assintomáticos com aPL persistentemente positivo não se beneficiam de aspirina na prevenção primária da trombose, apresentam baixa taxa de incidência de trombose aguda e desenvolvem eventos vasculares quando fatores de risco adicionais estão presentes. 